\title{
Crystal Structure and Characterization of Human Heavy-Chain Only Antibodies Reveals a Novel, Stable Dimeric Structure Similar to Monoclonal Antibodies \\ Soheila Bahmanjah ${ }^{1}$ \\ ${ }^{1}$ Merck \& Co. \\ soheila.bahmanjah@merck.com
}

\begin{abstract}
We report the novel crystal structure and characterization of symmetrical, homodimeric humanized heavy-chainonly antibodies or dimers ( $\mathrm{HC} 2 \mathrm{~s})$. HC2s were found to be significantly coexpressed and secreted along with mAbs from transient $\mathrm{CHO} \mathrm{HC} / \mathrm{LC}$ cotransfection, resulting in an unacceptable $\mathrm{mAb}$ developability attribute. Expression of full-length $\mathrm{HC} 2 \mathrm{~s}$ in the absence of LC followed by purification resulted in $\mathrm{HC} 2 \mathrm{~s}$ with high purity and thermal stability similar to conventional mAbs. The $\mathrm{VH}$ and $\mathrm{CH} 1$ portion of the heavy chain (or $\mathrm{Fd}$ ) was also efficiently expressed and yielded a stable, covalent, and reducible dimer (Fd2). Mutagenesis of all heavy chain cysteines involved in disulfide bond formation revealed that Fd2 intermolecular disulfide formation was similar to Fabs and elucidated requirements for $\mathrm{Fd} 2$ folding and expression. For one $\mathrm{HC} 2$, we solved the crystal structure of the $\mathrm{Fd} 2$ domain to $2.9 \AA$, revealing a highly symmetrical homodimer that is structurally similar to Fabs and is mediated by conserved (CH1) and variable (VH) contacts with all CDRs positioned outward for target binding. Interfacial dimer contacts revealed by the crystal structure were mutated for two $\mathrm{HC} 2 \mathrm{~s}$ and were found to dramatically affect $\mathrm{HC} 2$ formation while maintaining mAb bioactivity, offering a potential means to modulate novel $\mathrm{HC} 2$ formation through engineering. These findings indicate that human heavy-chain dimers can be secreted efficiently in the absence of light chains, may show good physicochemical properties and stability, are structurally similar to Fabs, offer insights into their mechanism of formation, and may be amenable as a novel therapeutic modality.
\end{abstract}

